\title{
BMJ Open Delayed diagnosis and treatment of pulmonary tuberculosis in high-burden countries: a systematic review protocol
}

\author{
Alvin Kuo Jing Teo, ${ }^{\oplus}$ Shweta R Singh, ${ }^{1}$ Kiesha Prem, ${ }^{1}$ Li Yang Hsu, ${ }^{1}$ Siyan $\mathrm{Yi}^{1,2}$
}

To cite: Teo AKJ, Singh SR, Prem K, et al. Delayed diagnosis and treatment of pulmonary tuberculosis in highburden countries: a systematic review protocol. BMJ Open 2019;9:e029807. doi:10.1136/ bmjopen-2019-029807

- Prepublication history and additional material for this paper are available online. To view these files, please visit the journal online (http://dx.doi. org/10.1136/bmjopen-2019029807).

Received 13 February 2019 Revised 5 June 2019 Accepted 12 June 2019

Check for updates

(C) Author(s) (or their employer(s)) 2019. Re-use permitted under CC BY-NC. No commercial re-use. See rights and permissions. Published by BMJ.

${ }^{1}$ Saw Swee Hock School of Public Health, National University of Singapore, Singapore

${ }^{2}$ Center for Population Health Research, KHANA, Phnom Penh, Cambodia

Correspondence to

Alvin Kuo Jing Teo; alvin.teo@aol.com

\begin{abstract}
Introduction Countries identified to bear the highest tuberculosis (TB) incidence account for approximately $85 \%$ of the global TB burden. TB is curable, yet nearly $40 \%$ of TB cases remained undiagnosed hence delaying treatment and perpetuating transmission. This systematic review aimed to review current evidence on factors associated with delayed diagnosis and treatment of TB in the high TB-burden countries.
\end{abstract}

Methods and analysis This systematic review will incorporate qualitative and observational study designs published between 2008 and 2018. Articles will be retrieved from major databases including PubMed, EMBASE, CINAHL and PsycINFO. Reference lists of key articles, including relevant systematic reviews and meta-analysis, will be screened for additional studies. Two independent reviewers will screen and select studies, extract data and assess the quality and risk of bias of each study. Study-specific estimates will be pooled by meta-analysis, and effect sizes will be presented as $\mathrm{OR}$ and their $95 \% \mathrm{Cl}$. Levels of heterogeneity will be evaluated using chi-square statistic $Q$ and $\mathrm{I}^{2}$. Publication bias will be assessed using forest plots and Egger's tests. Qualitative findings and sample quotes will be extracted. Textual references to the topics of interest will be retrieved and categorised using qualitative thematic analysis. We will triangulate quantitative and qualitative findings for a complete understanding of the reasons for delayed TB diagnosis and treatment. Results will be presented by geographical region.

Ethics and dissemination This study will be conducted based on published data. This systematic review may provide insights into the reasons for delayed TB diagnosis in highburden countries. These findings will also inform future research and key stakeholders in developing interventions to reach these undiagnosed cases effectively. Findings from this review will be published in a peer-reviewed journal.

PROSPERO registration number CRD42018107237

\section{INTRODUCTION}

Tuberculosis (TB) is a leading infectious cause of morbidity and mortality worldwide, accounting for 10 million new cases and 1.6 million deaths in 2017. ${ }^{1}$ The disease burden is disproportionately concentrated in low-income and middle-income countries, with over $95 \%$ TB deaths contributed by these regions. ${ }^{12}$ Many of these deaths are preventable through early diagnosis and treatment, yet, in 2017,

\section{Strengths and limitations of this study}

To the best of our knowledge, no published systematic review addresses delayed diagnosis and initiation of tuberculosis (TB) treatment in high-burden countries.

- This study will review and triangulate qualitative and quantitative findings to provide a more comprehensive and holistic view on the matter.

- We anticipate that this review might not be represented by all 30 countries in the high TB burden list due to the lack of evidence and research activities in a few countries.

- Another possible limitation may be the heterogeneity of the studies exploring the factors associated with delayed diagnosis and treatment of TB.

nearly $40 \%$ of TB cases remained undiagnosed globally. ${ }^{1}$ Limited access to healthcare, high treatment cost and the social stigma of TB have contributed to delayed detection and poor treatment uptake. ${ }^{3}$ Other risk factors such as poor living conditions and overcrowding further perpetuate the transmission of $\mathrm{TB}$ and infection, which, in turn, leads to social and economic insecurity. ${ }^{5}$ The financial burden of TB attributed by the loss of income, logistics and medical expenditures is high, with the total costs incurred amounting to 58\% and $39 \%$ of reported annual individuals and household income, respectively. ${ }^{6}$

Traditionally, TB cases are captured and passively notified when people with $\mathrm{TB}$ present themselves to a health facility. In recent years, a more proactive strategy to increase TB case detection, namely active case finding (ACF) has gained traction and is reported to be effective in promptly identifying people with $\mathrm{TB}^{7}$ Alongside passive case finding, the ACF strategy has been adopted by countries affected by the epidemic to reach people with TB effectively. ${ }^{8}$ Nevertheless, despite increased efforts to improve case detection, TB case finding remains a great challenge due to limited resources, geographical barriers and social stigma. ${ }^{10-12}$ 
Delays in TB diagnosis and care will increase the prevalence of infectious $\mathrm{TB}$, perpetuates greater transmission and ultimately impedes the global efforts to eliminate TB by $2030 .^{1314}$

Delays in diagnosis and detection have been identified as one of the key challenges to eliminate TB. ${ }^{15}$ A review of the literature found several published systematic reviews on factors associated with delayed TB diagnosis. Two systematic reviews focused primarily on reasons for delayed TB diagnosis in India. ${ }^{16}$ Two other reviews published in 2017 were specific to children and youth in sub-Saharan Africa and hard-to-reach populations such as migrants, refugees, prisoners, sex workers, people living with HIV, people who use drugs and homeless people. ${ }^{18} 19$ Methodologically, most reviews evaluated predominantly qualitative data, and reviewers have expressed limitations in reporting summary estimates due to great heterogeneity of the studies. ${ }^{1920}$ One review conducted in 2008 attempted to extract findings from quantitative studies, regardless if they met the criteria for a meta-analysis. ${ }^{21}$ These studies, though, were mostly conducted between the 1990s and early 2000s. ${ }^{21}$ With regard to geographical regions, a recent review investigated the factors associated with delayed diagnosis in the low-income and middle-income countries but included only observational study design, and no qualitative studies were synthesised. ${ }^{4}$ Also, a scoping review with a specific focus on the relationship between acceptability barrier (expectations from health providers/care process, attitudes of health providers and health beliefs) and delayed diagnosis of TB among high multidrug-resistant TB countries was recently published in 2016, but only qualitative studies were synthesised. ${ }^{22}$ Collectively, there are empirical evidence to associate various factors-sociodemographic, clinical, health system, economic and political-to delay in healthcare seeking and treatment of TB. However, delays in diagnosis and treatment exist to varying degrees across countries with a different population and a different burden of the disease. To our knowledge, no published systematic review addresses delayed diagnosis and initiation of TB treatment in countries bearing most of the global TB burden. There is also a lack of review that triangulates qualitative and quantitative findings to provide a more comprehensive and holistic view on the matter.

The WHO developed the concept of high-burden countries in 1998, and it was conceptualised to focus global efforts in countries where improvement is needed to curb the epidemic. The concept has since been widely used to rally political commitments, funding and interventions in these countries. ${ }^{23}$ There are currently three high-burden countries list for $\mathrm{TB}-\mathrm{TB}$, multidrug-resistant TB and TB/HIV-that are defined for 2016-2020. ${ }^{23}$ Each of this list accounts for approximately $85 \%$ of the global burden in their respective domains. Hence, it is pertinent to focus efforts on these high-burden countries to end TB globally by 2035 .

\section{Objective of systematic review}

This systematic review aimed to review current evidence on factors associated with delayed diagnosis and treatment of pulmonary TB in the high TB burden countries to inform the development of effective case finding and TB prevention interventions. We will focus on the delayed diagnosis of pulmonary TB regardless of the drug sensitivity or resistant status of the infecting TB pathogen.

\section{METHODS}

\section{Eligibility criteria}

We will consider studies that include people with TB, presumptive TB, key stakeholders involved in TB management and controls, the general and other populations that include, but not limited to, migrants and refugees, elderly, children, people living with HIV, prisoners and people with diabetes.

This review will include qualitative, observational studies (cross-sectional studies, case-control studies and cohort studies-both retrospective and prospective) conducted in the WHO high TB burden countries, namely Angola, Bangladesh, Brazil, Cambodia, Central African Republic, China, Congo, Democratic People's Republic of Korea, Democratic Republic of Congo, Ethiopia, India, Indonesia, Kenya, Lesotho, Liberia, Mozambique, Myanmar, Namibia, Nigeria, Pakistan, Papua New Guinea, the Philippines, Russian Federation, Sierra Leone, South Africa, Tanzania, Thailand, Vietnam, Zambia and Zimbabwe.

All studies that investigated factors associated with delayed TB diagnosis and initiation of treatment and published between 2008 and 2018 will be considered. These factors include demographics, socioeconomic, clinical, knowledge and attitude variables. As TB diagnosis criteria might differ from one country to another, we will regard all TB cases evaluated by a health facility that is equipped or linked to a laboratory with one of the diagnostic tools-smear microscopy, culture, GeneXpert and drug susceptibility test-and the presence of clinicians to make the final diagnosis as TB. We will exclude studies that evaluated self-reported TB cases if any. We will not impose limits on the studies by sample size. We will include the following primary outcomes-(1) patient delay: the time interval between the onset of symptoms and the first encounter with healthcare professionals; (2) health system/doctor/provider/diagnostic delay: the time interval between the first encounter with healthcare professionals and the diagnosis of pulmonary TB; (3) treatment delay: the time interval between $\mathrm{TB}$ diagnosis and the initiation of anti-TB treatment; and (4) total delay: the time interval between onset of symptoms and the initiation of anti-TB treatment.

We will exclude systematic reviews, meta-analyses, scoping reviews, intervention studies and non-English articles (except articles in Chinese), studies lacking key outcome data and/or unclear reporting of primary outcomes and publications in the form of letters, reviews, 
commentaries and editorials. The meta-analysis will only include studies with regression analysis as we will need the effect sizes of the factors associated with TB diagnosis and initiation of treatment. Relevant studies without regression analysis will only be reviewed in the narrative.

\section{Search strategy and information sources}

The search strategy aims to find published studies and will include three stages. First, a limited search of articles on PubMed and EMBASE, and an analysis of the index terms and keywords contained in the title and abstract will be undertaken. Appropriate free-text index terms, keywords, and Medical Subject Headings terms will be developed from the initial search. Using these identified search terms, a second search will be conducted across PubMed, EMBASE, CINAHL and PsycINFO using Boolean logic operators-AND and OR. A draft search strategy for EMBASE is appended in online supplementary file 1 . Third, the reference lists of key articles, including relevant systematic reviews and meta-analyses, will be screened for additional studies. In this review, we will consider studies published in English and Chinese between 2008 and 2018, inclusive.

\section{Study selection}

All identified citations will be exported into EndNote X8 (Clarivate Analytics, Philadelphia, Pennsylvania, USA) and duplicate will be removed. Subsequently, citations will be exported to Microsoft Excel Office 365 (Microsoft Corporation, Washington, DC, USA). Two independent reviewers will screen the titles and abstracts based on the inclusion criteria. Full-text articles of the selected citations will be retrieved and screened by two independent reviewers. We will exclude full-text articles that do not meet the inclusion criteria and include the reasons for these exclusions in the final review. Selected studies will be critically appraised, and data from each respective study will be extracted and synthesised. The results of the search will be presented in accordance with the Preferred Reporting Items for Systematic Review and Meta-Analysis (PRISMA) guideline. We will seek the opinion of a third reviewer should any disagreements arise between the two primary reviewers.

\section{Quality assessment}

Selected studies will be critically assessed by two independent reviewers using the Newcastle-Ottawa Scale to assess the quality of non-randomised studies (cross-sectional studies, case-control studies and cohort studies) in meta-analyses. ${ }^{24}$ We will assess qualitative studies using the Critical Appraisal Skills Programme (CASP) tool..$^{25}{ }^{26}$ In addition, we will grade the final synthesised qualitative findings according to the ConQual approach to rate the level of confidence and certainty of the findings. ${ }^{27}$ Synthesised findings will be presented in a Summary of Findings table, which will include information on the type of research, dependability, credibility, ConQual score and comments on the findings, if any. Grading of the dependability and credibility of synthesised findings will be done using the Joanna Briggs Institute Summary of Findings Tables. ${ }^{28}$ The Summary of Findings table will also include the systematic review title, population, phenomena of interest and the context. Any disagreements will be resolved through discussion with a third reviewer.

\section{Data extraction and management}

Quantitative and qualitative data will be extracted independently by two researchers. We will develop a standardised form to record study (eg, year, study design, location and sample size) and participants' characteristics (eg, study respondents, median/mean age, sex and the duration of delay, if available), primary outcome measures (patient delay, doctor/health system delay and treatment delay), main findings (factors associated with delays) and study quality scores. In the event where information regarding the selected studies are lacking, we will contact the respective authors and request for the information.

\section{Data synthesis and analyses}

We will report study settings, countries and sample size by study design. A narrative synthesis of the independent variables will be generated. We anticipate that there will be significant variations in the factors associated with delayed diagnosis. Howbeit, we will seek to estimate the effect of factors associated with a delayed diagnosis and treatment if data are available in two or more studies. Data will be pooled by meta-analysis using RevMan V.5 (The Cochrane Collaboration, Oxford, UK) and R (R Foundation for Statistical Computing, Vienna, Austria). We will present effect sizes as OR and their 95\% CI. Levels of heterogeneity will be quantified using chi-square statistic $\mathrm{Q}$ and $\mathrm{I}^{2}$. If considerable heterogeneity will be detected, a subgroup analysis on smear status and the geographical region will be performed to assess its source. Findings will be presented in forest plot and narrative form if statistical pooling is not feasible. Publication bias will be assessed using forest plots and Egger's tests. ${ }^{29}$ For qualitative studies, findings and sample quotes reported will be extracted verbatim. We will annotate and analyse the extracted data using NVIVO by QSR International. Textual references to topics of interest will be retrieved and categorised using qualitative thematic analysis. Two authors will code the data independently, and discrepancies will be discussed to standardise code definitions. We will examine codes in details for subthemes and patterns. We will synthesise these data with quantitative findings for a complete understanding of the reasons for delayed TB diagnosis and treatment. We aim to perform gender-based analyses of the factors associated with delayed diagnosis and treatment of TB and present results by geographical region that the high-burden countries represent.

\section{Presentation and reporting of results}

A flow chart representing each stage of the studies' selection process will be presented as per the PRISMA guidelines. We will present factors associated with patient delay, health 
system/doctor/provider/diagnostic delay and treatment delay among high TB burden countries using tables and forest plots. Risk of bias scores for methodological quality of studies included in this review will be tabulated together with comments supporting the decisions.

\section{Patient and public involvement}

Patients and members of the public were not involved in the design and development of this research. The findings from this review will be published and shared in conference presentation.

\section{DISCUSSION}

To our knowledge, this systematic review will be the first to focus on countries with a high TB burden. This protocol was developed using validated tools and processes, yet, there are several noteworthy limitations. First, we might miss studies that are relevant to this review. We strive to minimise this issue by working with a librarian on the search strategy and assessing the reference list of similar reviews that have been published. Second, we anticipate that this review might not be represented by all 30 countries in the high TB burden list due to the lack of evidence and research activities in several countries. Therefore, it may be difficult to conclude universally for all high TB burden countries.

Nevertheless, we hope that this review may highlight gaps in knowledge and provide insights into the reasons for delayed TB diagnoses in high-burden countries. These findings will also inform future research and key stakeholders in developing interventions to reach these undiagnosed cases effectively.

Contributors AKJT and SRS designed the study and will acquire data. AKJT, SRS and KP analysed and interpreted the data. AKJT and SRS wrote the initial draft of the manuscript. AKJT, SRS, KP, LYH and SY critically reviewed the final manuscript. All authors approved the final manuscript.

Funding The authors have not declared a specific grant for this research from any funding agency in the public, commercial or not-for-profit sectors.

Competing interests None declared.

Patient consent for publication Not required.

Provenance and peer review Not commissioned; externally peer reviewed.

Open access This is an open access article distributed in accordance with the Creative Commons Attribution Non Commercial (CC BY-NC 4.0) license, which permits others to distribute, remix, adapt, build upon this work non-commercially, and license their derivative works on different terms, provided the original work is properly cited, appropriate credit is given, any changes made indicated, and the use is non-commercial. See: http://creativecommons.org/licenses/by-nc/4.0/.

\section{REFERENCES}

1. World Health Organization. Global tuberculosis report 2018. Geneva: World Health Organization, 2018.

2. Kyu HH, Maddison ER, Henry NJ, et al. The global burden of tuberculosis: results from the Global Burden of Disease Study 2015. Lancet Infect Dis 2017:1-24.

3. Courtwright A, Turner AN. Tuberculosis and stigmatization: pathways and interventions. Public Health Rep 2010;125 Suppl 4(Suppl 4):34-42.

4. Getnet F, Demissie M, Assefa N, et al. Delay in diagnosis of pulmonary tuberculosis in low-and middle-income settings: systematic review and meta-analysis. BMC Pulm Med 2017;17:202.
5. Laxminarayan R, Klein E, Dye C, et al. Economic benefit of tuberculosis control. Policy Research Working Paper: World Bank, 2007. (Policy Research Working Paper). Report No.: 4925.

6. Tanimura T, Jaramillo E, Weil D, et al. Financial burden for tuberculosis patients in low- and middle-income countries: a systematic review. Eur Respir J 2014;43:1763-75.

7. Mhimbira FA, Cuevas LE, Dacombe R, et al. Interventions to increase tuberculosis case detection at primary healthcare or community-level services. Cochrane Database Syst Rev 2017;11:CD011432.

8. Eang MT, Satha P, Yadav RP, et al. Early detection of tuberculosis through community-based active case finding in Cambodia. BMC Public Health 2012;12:1-9.

9. Morishita F, Eang MT, Nishikiori N, et al. Increased case notification through active case finding of tuberculosis among household and neighbourhood contacts in Cambodia. PLoS One 2016;11:e0150405.

10. Kranzer K, Afnan-Holmes $\mathrm{H}$, Tomlin $\mathrm{K}$, et al. The benefits to communities and individuals of screening for active tuberculosis disease: a systematic review. Int J Tuberc Lung Dis 2013;17:432-46.

11. Murray EJ, Bond VA, Marais BJ, et al. High levels of vulnerability and anticipated stigma reduce the impetus for tuberculosis diagnosis in Cape Town, South Africa. Health Policy Plan 2013;28:410-8.

12. Yaesoubi R, Cohen T. Identifying dynamic tuberculosis casefinding policies for HIV/TB coepidemics. Proc Natl Acad Sci U S A 2013;110:9457-62.

13. Golub JE, Bur S, Cronin WA, et al. Delayed tuberculosis diagnosis and tuberculosis transmission. Int J Tuberc Lung Dis 2006;10:24-30.

14. The Global Fund to Fight AIDS, Tuberculosis and Malaria. Make a global priority of finding missing cases of tuberculosis [Internet]. 2017 https://www.theglobalfund.org/en/blog/2017-10-10-make-aglobal-priority-of-finding-missing-cases-of-tuberculosis/ (Accessed 2018 Jul 11).

15. Jassal MS, Bishai WR. Epidemiology and challenges to the elimination of global tuberculosis. Clin Infect Dis 2010;50(0 3):S156-S164.

16. Sreeramareddy CT, Qin ZZ, Satyanarayana S, et al. Delays in diagnosis and treatment of pulmonary tuberculosis in India: a systematic review. Int J Tuberc Lung Dis 2014;18:255-66.

17. Samal J. Health seeking behaviour among tuberculosis patients in India: a systematic review. J Clin Diagn Res 2016;10:LE01-6.

18. de Vries SG, Cremers AL, Heuvelings CC, et al. Barriers and facilitators to the uptake of tuberculosis diagnostic and treatment services by hard-to-reach populations in countries of low and medium tuberculosis incidence: a systematic review of qualitative literature. Lancet Infect Dis 2017;17:e128-43.

19. Sullivan BJ, Esmaili BE, Cunningham CK. Barriers to initiating tuberculosis treatment in sub-Saharan Africa: a systematic review focused on children and youth. Glob Health Action 2017;10:1290317.

20. Sreeramareddy CT, Panduru KV, Menten J, et al. Time delays in diagnosis of pulmonary tuberculosis: a systematic review of literature. BMC Infect Dis 2009;9:91.

21. Storla DG, Yimer S, Bjune GA. A systematic review of delay in the diagnosis and treatment of tuberculosis. BMC Public Health 2008;8:15.

22. Barnabishvili M, Ulrichs T, Waldherr R. Role of acceptability barriers in delayed diagnosis of Tuberculosis: Literature review from high burden countries. Acta Trop 2016;161:106-13.

23. World Health Organization. Use of high burden country lists for TB by WHO in the post-2015 era. Geneva: World Health Organization, 2015.

24. Wells G, Shea B, O'connell D, et al. The Newcastle-Ottawa Scale (NOS) for assessing the quality of nonrandomised studies in meta-analyses [Internet]. http://www.ohri.ca/programs/clinical_ epidemiology/oxford.asp (Accessed 2019 Jan 26).

25. CASP - Critical Appraisal Skills Programme. CASP Checklists [Internet]. https://casp-uk.net/casp-tools-checklists/ (Accessed 2019 Jan 26).

26. Noyes J, Booth A, Flemming K, et al. Cochrane Qualitative and Implementation Methods Group guidance series-paper 3: methods for assessing methodological limitations, data extraction and synthesis, and confidence in synthesized qualitative findings. $J$ Clin Epidemiol 2018;97:49-58.

27. Munn Z, Porritt K, Lockwood C, et al. Establishing confidence in the output of qualitative research synthesis: the ConQual approach. BMC Med Res Methodol 2014;14:108.

28. The Joanna Briggs Institute Level of Evidence and Grades of Recommendation Working Party. Summary of findings tables for Joanna Briggs Institute Systematic Reviews. Adelaide: The Joanna Briggs Institute, 2016.

29. Egger M, Davey Smith G, Schneider M, et al. Bias in meta-analysis detected by a simple, graphical test. BMJ 1997;315:629-34. 